Article

\title{
Design of 3D Structure Membrane for the Increased Sensitivity in Enzyme Linked Immunosorbent Assay (mELISA)
}

\author{
Anna Go $\mathbb{D}$, Young Ju Park and Min-Ho Lee *(D) \\ School of Integrative Engineering, Chung-Ang University, 84 Heukseok-ro, Dongjak-gu, Seoul 06974, Korea; \\ anna@itsannastyle.com (A.G.); qkryju93@hanmail.net (Y.J.P.) \\ * Correspondence: mhlee7@cau.ac.kr; Tel.: +82-2-820-5503
}

Received: 30 August 2019; Accepted: 1 October 2019; Published: 5 October 2019

check for updates

Featured Application: The developed plastic membrane can be implemented in an automatic pretreatment device for immunoassays. The plastic membrane can be easily coupled with a robotic arm. With a simple up-and-down motion, the pretreatment steps of immunoassays, including washing and conjugation, can be easily prepared.

\begin{abstract}
The Enzyme Linked Immunosorbent Assay (ELISA) technique has been widely used for the identification and quantification of biochemical markers. The typical ELISA requires a number of washing steps to eliminate the unbound proteins which sometimes cause the desorption of protein due to their weak bonding between protein and well plate. In this study, we have developed a meshed type of plastic membrane in order to increase the reliable binding efficiency between proteins and the membrane surface, and to provide easy steps of washing. The use of our developed solid membrane has significantly increased the binding capacity of the biomolecules because this membrane ELISA (mELISA) provides 3D binding surfaces which increases the surface area when compared to the conventional 2D surface well plate. The columns were pretreated to form a self-assembled layer (SAM) on the surface for the stable conjugation of a target antibody. The SAM-coated membranes could be stored for one month without any further deterioration of stability. The measured optical density (O.D.) shows a 1.2-fold increase in IgG antigen $(25 \mu \mathrm{g} / \mathrm{mL})$ from the plastic membrane as compared with the conventional ELISA method. The concentrations of thyroid stimulating hormone were also monitored using the mELISA method and it shows good linearity against the concentrations.
\end{abstract}

Keywords: meshed membrane; ELISA 2; 3D structure; TSH hormone

\section{Introduction}

The enzyme-linked immunosorbent assay (ELISA) is a widely used technique that utilizes an enzyme linked antibody and substrate to identify the target antigens. Solid supports such as membrane and polystyrene are commonly available in biomedical applications. There are two types of interest for membrane design, namely inorganic and organic membranes. Inorganic membranes are common supports that could increase the antigen-antibody reactions by increasing the surface area and performing a capillary action. Organic membranes have generally been used and applied commercially for biosensors. The commonly available membrane-based biosensor systems include a filtration function such as nitrocellulose [1], nanofiber, cellulose [2], and nylon [3]. The other substrates based on organic membranes include polymer components, polyethersulfone [4], polydimethylsiloxane (PDMS) [3], polypropylene, polylactic acid (PLA), and polycarbonate [5]. Polymer substrates are the type of immobilization column generally used for the ELISA system as they provide a low fabrication process cost and ease of use. 
These membranes were immobilized between solid supports and biomolecules by either adsorption or covalent bonding. The typical ELISA requires a number of washing steps to remove the unbound proteins, but these can also cause the desorption of protein due to their weak binding [6]. Because of the weak binding property, immobilization methods have been studied with the aim of improving the bonding of the biomolecule on the solid surface covalently [7]. By contrast, solid surface and biological material can be combined with the highly sophisticated self-assembled layer (SAM). The SAM has been studied by many different and interdependent surface science techniques. Surface treatment has involved treatment with gamma-ray [8] and oxygen plasma for increasing the binding ability between membranes and biomolecules. The ability of ELISA explains the use of an enhanced molecule absorption area for a greater bonding area between the solid support and antibody. The 3D printed fabricated membrane provided enhanced molecule detection sensitivity as compared to that of the conventional ELISA [9]. In addition, gold nanoparticles (AuNPs) were used as probes of the antibody-conjugated with HRP (horseradish peroxidase) in order to enhance the detection of the area of breast cancer biomarker [10].

In this study, we have developed the 3D plastic column type solid phase or membrane structure, on which conjugated antibodies and antigens react. Using this new column type plastic membrane, we performed a typical ELISA by moving and dipping the membrane itself into different wells instead of transferring and washing buffers in the stationary bottom surface, which are typical steps of the ELISA. Interestingly, an improvement in measured signals could be achieved when we used the 3D membrane. In addition, we have applied a covalent bonding method between the antibody and solid surface of the membrane, which accounts for increased optical density (O.D.) results in corresponding conditions. For the immune-reaction test, we performed a characterization test using IgG antigen.

For the sensitivity-related test, we have chosen and performed the quantification of thyroid stimulating hormone (TSH). TSH is one of the most significant kinds of hormone and regulates the release of the metabolic hormones such as T3 (triiodothyronine) and T4 (thyroxine). In addition, because of their low concentrations present in serum, it is more difficult to detect without using highly sensitive sensors. In this study, we demonstrated that the developed mELISA method could provide a highly sensitive detection in low levels of TSH $(0.01 \sim 0.1 \mathrm{ng} / \mathrm{mL})$, which is mostly difficult to measure when using the conventional ELISA.

\section{Materials and Methods}

\subsection{Materials}

Sodium phosphate dibasic (Na2HPO, S7907), sodium phosphate monobasic dihydrate (NaH2PO4-H2O, 13472-35-0), glutaraldehyde solution (G6403), (3-aminopropyl) triethoxysilane (APTES, 281788), and potassium hydroxide $(\mathrm{KOH}, 221473)$ were purchased from Sigma Aldrich. Anti-mouse IgG (whole molecule) antibody produced in goat (M8642), IgG from mouse serum (I5381), Anti-mouse IgG (whole molecule)-Biotin antibody produced in goat (B7264), Bovine Serum Albumin (BSA, A7030), and Streptavidin-Peroxidase from Streptomyces avidinii (SA-HRP, S5512) were all sourced from Sigma Aldrich. Further, one-Step Ultra TMB-ELISA (34028) reagents were purchased from ThermoFisher Scientific and used as received without purification. A 4-(2-hydroxyethyl) -1-piperazineethanesulfonic acid (HEPES) solution was purchased from Biosesang (H2003-1). Thyroid stimulating hormone (TSH) was purchased from Fitzgerald (TSH protein, 30-1237). Anti-TSH capture antibody was purchased from Abcam (Anti-TSH antibody, 11E4). Anti-TSH detection antibody was purchased from Abcore (TSH detection Ab-biotin, AC15-0144-0).

\subsection{Design of Membrane}

The plastic membrane has a cylindrical shape with dimension of $0.35 \times 1.2 \mathrm{~cm}$ (radius $\times$ height). Its three-dimensional structure provides a greater surface reaction area than a conventional ELISA plate which uses only the bottom surface for the immobilization of antibodies. When calculated using 
a SOLID WORKS program, the surface area of the developed membrane is calculated to be $128.96 \mathrm{~mm}^{2}$ whereas the surface area of typical ELISA plate is $80.384 \mathrm{~mm}^{2}$, which brings a 1.6 -fold increase of the surface area (Figure 1). The developed meshed type MEMBRANE structure is made of polystyrene and it was designed and fabricated using a metallic mold for further production.

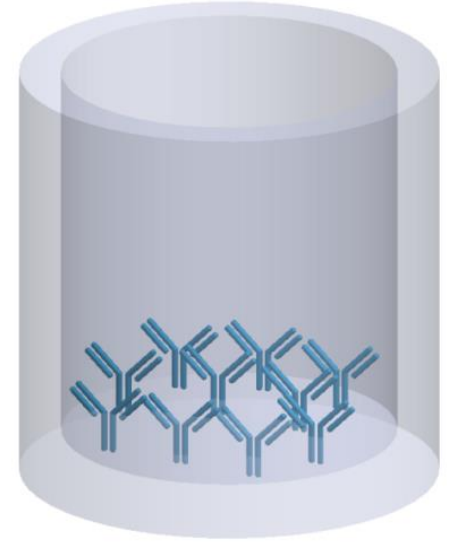

(a)

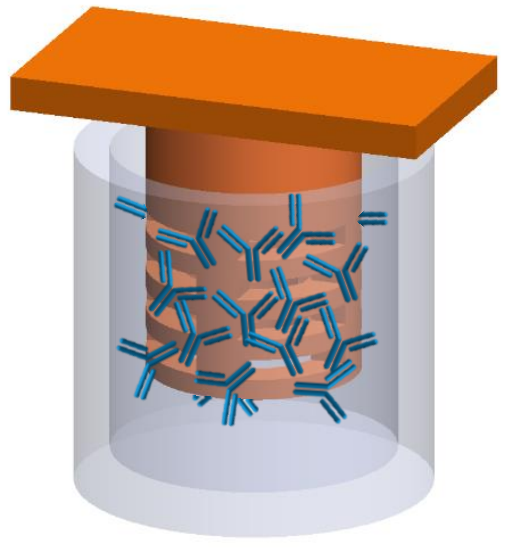

(b)

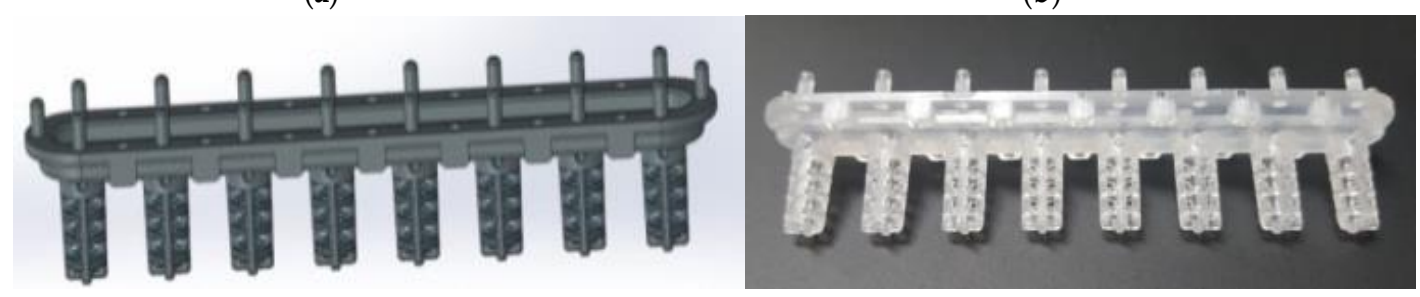

(c)

Figure 1. Schematic of plastic membrane design for this study. (a) Most antibodies are conjugated on the bottom surface in a conventional ELISA well plate, (b) the fabricated membrane increases the surface area where antibodies are conjugated, and (c) a designed and fabricated meshed type plastic membrane.

\subsection{Surface Modification of Membrane}

The surfaces of the membranes were treated with potassium hydroxide, 3-aminopropyltriethoxysilane (3-APTES), and glutaraldehyde (GA) for the stable formation of a self-assembly monolayer (SAM) on the surface. The SAM process involved three steps. First, the membranes were etched with $30 \%$ $\mathrm{KOH}$ solution (in distilled water) to form -OH groups on the surface. Then, they were reacted with $1 \%$ 3 -APTES in 5\% EtOH for the formation of amine groups. Finally, the surfaces were then modified with $1 \%$ glutaraldehyde (GA) in phosphate buffer solution. Antibody proteins were immobilized for the immuno-reaction with antigen proteins.

\subsection{Calibration of IgG and TSH Antigen}

In terms of the overall processes of ELISA, they are composed of four basic steps: the immobilization of capture antibodies, blocking and washing, detection of target analytes, and optical density of analytes. The first step is the immobilization of capture antibodies on the surface of membranes. We used mouse IgG capture antibody $(10 \mu \mathrm{g} / \mathrm{mL}$ in $0.1 \mathrm{M}$ phosphate buffered saline (PBS)) for the optimization. Then, for the immobilization of capture antibody process, the membrane was incubated overnight at room-temperature. After washing with PBS, blocking solution ( $5 \%$ casein in $0.1 \mathrm{M} \mathrm{PBS)}$ was injected into the well for $60 \mathrm{~min}$ at the incubator to block all non-immobilized surface-binding sites.

The membrane was then rinsed with washing buffer ( $0.05 \%$ Tween 20 in $0.1 \mathrm{M}$ PBS) followed by a reaction with capture antibody at $37^{\circ} \mathrm{C}$ for $30 \mathrm{~min}$ and by additional washings. For the calibration, 
the concentration of serially diluted IgG antigens were prepared at $0.1,1,10,100$, and $1000 \mathrm{ng} / \mathrm{mL}$ in PBS, respectively. As a negative control, PBS solution was prepared without the presence of IgG antigen. After extensive washing, IgG antigen was reacted with $1 \mu \mathrm{g} / \mathrm{mL}$ detection IgG antibody (in $100 \mu \mathrm{g} / \mathrm{mL}$ BSA, $10 \mathrm{mM}$ HEPES (pH 7.4), $10 \mathrm{mM} \mathrm{NaCl}$, and $0.05 \%$ Tween-20 in $0.1 \mathrm{M} \mathrm{PBS}$ ) at $37^{\circ} \mathrm{C}$ for $30 \mathrm{~min}$. After another washing process, SA-HRP (1:5000 in 0.1M PBS) was conjugated to the detection antibody which was conjugated with IgG antigen. After all the process, the plate was incubated with TMB substrate at room temperature for $15 \mathrm{~min}$, and the absorbance was measured at $650 \mathrm{~nm}$ using an ELISA plate reader.

For TSH calibration, all the steps were performed using TSH antigen, capture antibody, and detection antibody instead of IgG antigen and antibody proteins.

\section{Results and Discussion}

The optimization of the conditions of surface treatment for fabricated membranes is especially important in improving the efficiency of adhesion between solid phases and biomolecules. The adhesion ability of a solid surface depends on many factors such as the $\mathrm{pH}$ and concentration of the modified buffer. To find out the optimal conditions for measurement, we performed a calibration of IgG antigen with varying incubation times, concentration of chemicals, and on different days. Figure 2 shows the changes of slope and linearity of the calibration according to the reaction incubation time with 3-APTES. The optimized incubation time with 3-APTES treatment is measured to be $120 \mathrm{~min}$ as can be seen in Figure 2a. The results show that the optical density with an 3-APTES reaction time less than $90 \mathrm{~min}$ was decreased by 1.5 times at $50 \mathrm{ng} / \mathrm{mL} \mathrm{IgG}$ antigen as compared with more than $90 \mathrm{~min}$. In terms of the low concentration range, i.e., below $40 \mathrm{ng} / \mathrm{mL}$, which is the main range concerning IgG, the calibration results showed better linearity than results with other incubation times. The linearity changes against concentrations with varying $\mathrm{pH}$ of GA solution in $0.1 \mathrm{M}$ phosphate buffer were measured by optical density (Figure $2 b, c$ ). The GA solution of $0.5 \%, 1 \%$, and $2 \%$ GA containing each concentration in phosphate buffer and $2 \%$ GA solution showed the maximum difference between $0 \mathrm{ng} / \mathrm{mL}$ and $100 \mathrm{ng} / \mathrm{mL}$ of antigen concentration (Figure $2 \mathrm{~b}$ ). As can be seen in Figure $2 \mathrm{c}$, the calibration linearity based on optical density was measured to be similar in $\mathrm{pH} 6.2$ and 7.2 of phosphate buffer solution. Figure $2 \mathrm{~d}$ shows the changes in calibration linearity during a four-week period. Five separate plastic membranes underwent surface pretreatment and were then stored in $\mathrm{EtOH}$ solution. Each of the five membranes was tested independently using the ELISA process with a period of one week. Interestingly, the membrane stored for only a day showed a higher intensity as compared to those stored for a longer period of time which showed no significant difference in optical densities.

The aforementioned surface treatments were applied to a plastic membrane and the optical density results of the IgG interaction were obtained before they were compared with those obtained using a typical ELISA. The optical density level of the ELISA system using a hybrid interface shows a 1.67 -fold increased binding ability at $100 \mathrm{ng} / \mathrm{mL}$ in the presence of IgG antigen as compared with the conventional ELISA. In addition, the developed membrane could detect as low as $0.15625 \mathrm{ng} / \mathrm{mL}$ of IgG antigen resulting in increases of 1.75-fold over the traditional ELISA. As shown in Figure 3a, the meshed polystyrene (PS) membrane increases linearly as the concentration of IgG increases, especially in the case of over $50 \mathrm{ng} / \mathrm{mL}$ of antigen, by more than traditional ELISA. The PS membrane ELISA method increased the reaction surface area due to its structural advantage and thus increased the optical density accordingly. Figure $3 \mathrm{~b}$ shows the effect of such materials as polypropylene (PP) and polymethyl methacrylate (PMMA) on the optical density of the ELISA system. In addition, the structure is made with machining in order to have a bar-shape and size of $0.35 \times 1.2 \mathrm{~cm}(\mathrm{r} \times 1)$. The bar-shape membrane was also treated with optimal chemicals as $\mathrm{KOH}$, APTES, and GA prior to conjugation with antibodies. They were measured using spectrophotometer to find out optical densities against different concentrations of antigens. As can be seen in Figure 3b, although the bar-shaped membrane showed lower optical density levels than those of the meshed shaped membrane, both of their slopes 
against antigen concentration showed good linearity. Overall, the structure shape played the main role in increasing the area of reaction which determined the amount of overall reactions.

Finally, for the clinical feasibility test, we have applied our membrane to measure the optical density changes against TSH concentrations. As can be seen in Figure 4, we could measure the differentiable optical density signal of TSH as low as $0.001 \mathrm{ng} / \mathrm{mL}$ which would not be in the measurable range of conventional ELISA. In general, the detection of TSH in a low range needs a highly sensitive detection modality. Furthermore, the process of labeling in order to increase its sensitivity should be carefully considered.

In this study, we could detect TSH antigen as low as $0.026 \mathrm{ng} / \mathrm{mL}$ using the normal ELISA. However, when we use our developed ELISA method using the plastic membrane, the observed detection limit was measured to be $0.001 \mathrm{ng} / \mathrm{mL}$. This sensitivity is comparable to other relatively high technology and luminescence based detection methods [11].

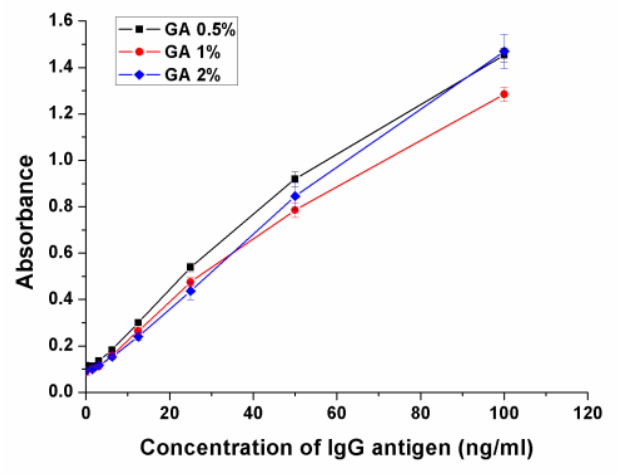

(a)

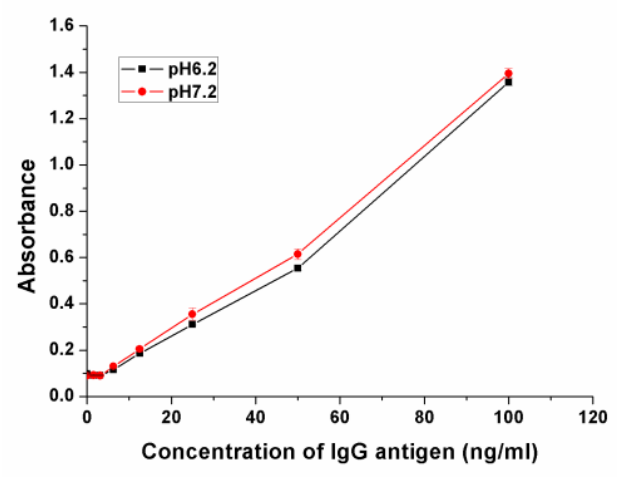

(c)

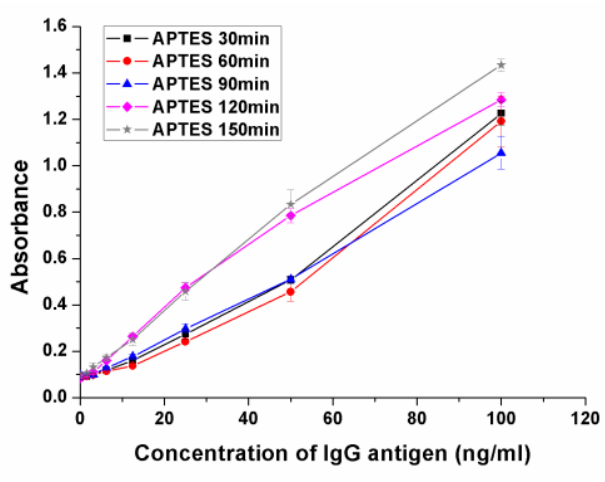

(b)

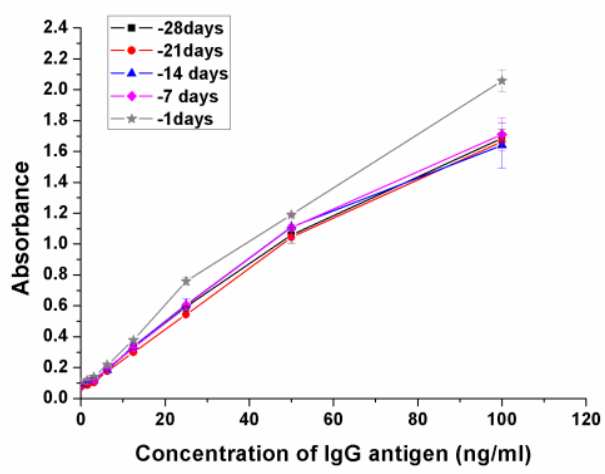

(d)

Figure 2. Changes in calibration linearity against different concentrations of IgG. Under conditions of (a) APTES incubation times of 30 150 min, (b) the amount of Glutaraldehyde, (c) pH of 6.2 and 7.2, and (d) different storage days from one to 28 days. 


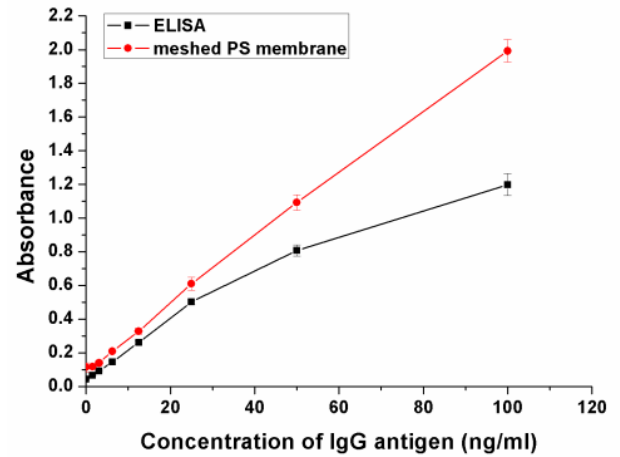

(a)

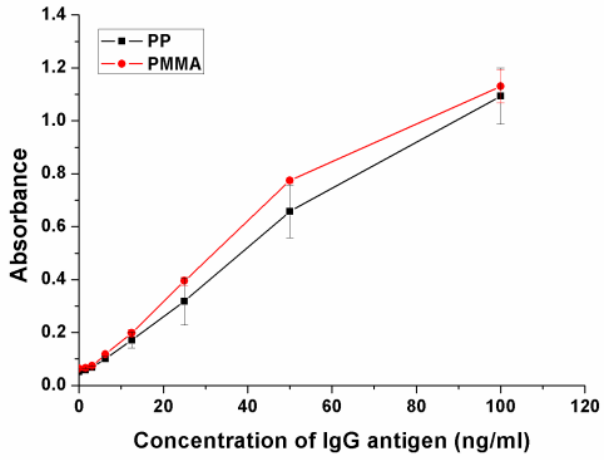

(b)

Figure 3. (a) Comparison between the conventional ELISA (black line) and meshed PS membrane type ELISA (red line) against a concentration of IgG antigens and (b) the effect of different materials on optical density.

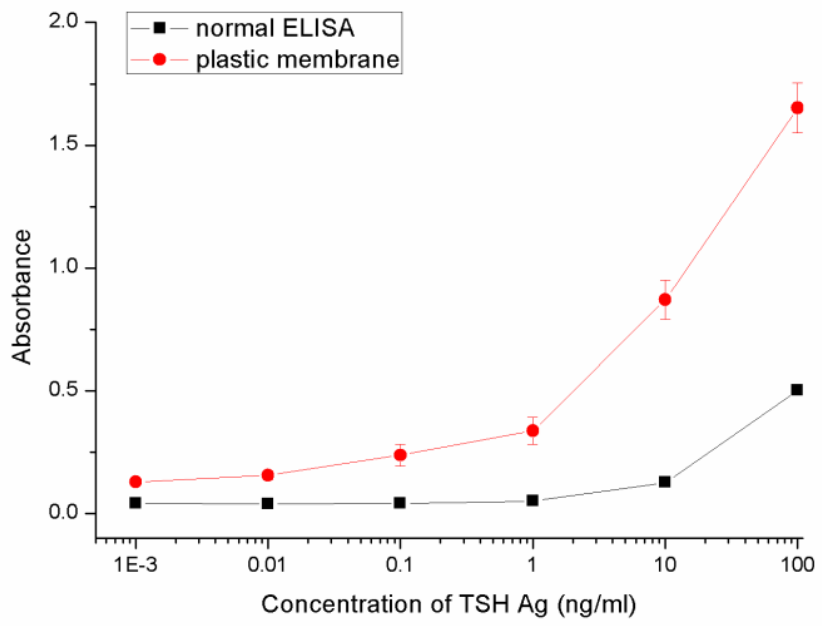

Figure 4. The optical density-based calibration of TSH antigen using a membrane as a platform for the immobilization of antibodies.

\section{Conclusions}

In this study, we have proposed a simplified ELISA methodology concept that includes a movable plastic membrane platform for the immobilization of capture antibodies and for the antigen-antibody interactions. Interestingly, the detectable ranges were considerably increased with an acceptable linearity, and both sensitivity and intensity were amplified as well. Due to its structural advantages, more antibodies were immobilized on the treated plastic surface, resulting in an enhancement of the possibilities of the reaction with target antigens as compared to the methodologies used in conventional ELISA system. The movable membrane type could also be easily integrated with an automated system which moves the membrane against the fixed solution wells instead of a liquid handling apparatus, as in most currently available systems for pretreatment.

Author Contributions: Conceptualization, A.G. and M.-H.L.; methodology, A.G. and M.-H.L.; resources, M.-H.L.; data curation, A.G. and Y.J.P.; writing—original draft preparation, A.G.; writing-review and editing, M.-H.L.; supervision, M.-H.L.; funding acquisition, M.-H.L.

Funding: "This research was funded by Ministry of Trade, Industry, and Energy grant number 10062995" and "This work was also funded by Chung-Ang University Research Grants in 2019".

Conflicts of Interest: The authors declare no conflict of interest. 


\section{References}

1. Abdel-Hamid, I.; Atanasov, P.; Ghindilis, A.L.; Wilkins, E. Development of a flow-through immunoassay system. Sens. Actuators B: Chem. 1998, 49, 202-210. [CrossRef]

2. Al-Kadhemy, M.F.H.; Saeed, A.A.; Khaleel, R.I.; Al-Nuaimi, F.J.K. Effect of gamma ray on optical characteristics of (pmma/ps) polymer blends. J. Theor. Appl. Phys. 2017, 11, 201-207. [CrossRef]

3. Campbell, G.A.; Medina, M.B.; Mutharasan, R. Detection of staphylococcus enterotoxin B at picogram levels using piezoelectric-excited millimeter-sized cantilever sensors. Sens. Actuators B: Chem. 2007, 126, 354-360. [CrossRef]

4. Fischer, L.M.; Wright, V.A.; Guthy, C.; Yang, N.; McDermott, M.T.; Buriak, J.M.; Evoy, S. Specific detection of proteins using nanomechanical resonators. Sens. Actuators B: Chem. 2008, 134, 613-617. [CrossRef]

5. Fung, Y.S.; Wong, Y.Y. Self-assembled monolayers as the coating in a quartz piezoelectric crystal immunosensor to detect salmonella in aqueous solution. Anal. Chem. 2001, 73, 5302-5309. [CrossRef] [PubMed]

6. Hong, Y.; Berrang, M.E.; Liu, T.; Hofacre, C.L.; Sanchez, S.; Wang, L.; Maurer, J.J. Rapid detection of campylobacter coli, c. Jejuni, and salmonella enterica on poultry carcasses by using pcr-enzyme-linked immunosorbent assay. Appl. Environ. Microbiol. 2003, 69, 3492-3499. [CrossRef] [PubMed]

7. Kim, J.-H.; Yoon, J.-Y. Protein adsorption on polymer particles. In Encyclopedia of Surface and Colloid Science; Marcel Dekker, Inc.: New York, NY, USA, 2002; Volume 1, p. 4373.

8. Park, I.-S.; Kim, N. Thiolated salmonella antibody immobilization onto the gold surface of piezoelectric quartz crystal. Biosens. Bioelectron. 1998, 13, 1091-1097. [CrossRef]

9. Singh, H.; Shimojima, M.; Fukushi, S.; Le Van, A.; Sugamata, M.; Yang, M. Increased sensitivity of 3d-well enzyme-linked immunosorbent assay (ELISA) for infectious disease detection using 3D-printing fabrication technology. Biomed. Mater. Eng. 2015, 26, S45-S53. [CrossRef] [PubMed]

10. Sheehan, M.T. Biochemical testing of the thyroid: TSH is the best and, oftentimes, only test needed - a review for primary care. Clin. Med. Res. 2016, 14, 83-92. [CrossRef] [PubMed]

11. Sarkar, R. TSH comparison between chemiluminescence (Architect) and electrochemiluminescence (Cobas) immunoassays: An indian population perspective. Indian J. Clin. Biochem. 2014, 29, 189-195. [CrossRef] [PubMed] 